\title{
FLUID-STRUCTURE INTERACTION AND TRANSIENT CAVITATION TESTS IN A T-PIECE PIPE
}

\author{
ARRIS S TIJSSELING \\ ALAN E VARDY \\ Department of Mathematics and Computer Science \\ Civil Engineering Division \\ Eindhoven University of Technology \\ University of Dundee \\ P.O. Box 513, 5600 MB Eindhoven \\ Dundee DD1 4HN \\ The Netherlands \\ United Kingdom \\ e-mail: a.s.tijsseling@tue.nl \\ e-mail: a.e.vardy@dundee.ac.uk
}

\begin{abstract}
A physical model featuring the combined phenomena of fluid-structure interaction (FSI) and vaporous cavitation is presented. The model is a closed, water-filled, T-shaped, laboratory pipe system, where the Tjunction and the three closed ends are potential locations for strong FSI coupling and/or column separation. The system is suspended horizontally on long steel wires and is excited through external impact. The impact force and the static pressure of the liquid control the severity of the cavitation phenomenon.
\end{abstract}

Keywords: Water hammer; Fluid-structure interaction (FSI); Vaporous cavitation; Column separation 


\section{Introduction}

Pressure variations in a water-hammer incident can cause low absolute pressures that enable vaporous and/or gaseous cavities to form. Sometimes, at specific locations such as closed ends, tees, knees and high points, the cavity can occupy the entire pipe cross-section, thereby separating adjacent regions of fully-liquid flow. The separated columns subsequently behave semi-independently and, in some circumstances, can induce a rapid collapse of the void, resulting in a sudden, large pressure rise. The steepness of wave fronts travelling away from the point of collapse can make it likely that less-restrained parts of the pipe system will move significantly as a result of unbalanced pressure forces. This paper reports an experimental study of these phenomena in a T-shaped pipe system.

Reviews by Tijsseling (1996) and Wiggert and Tijsseling (2001) reveal that both FSI experiments in pipe systems with branches and FSI experiments with cavitation are rare. In that sense, the present study is entirely new: it combines FSI and cavitation in a system with a branch. The study builds on previous work. Vardy and Fan (1989) measured the transient vibration of a straight, liquid-filled pipe and they analysed FSI in the time domain. Zhang et al. (1999) considered the free vibration of the same pipe with measurement and analysis in the frequency domain. L-shaped and T-shaped systems have been the subject of study in (Tijsseling et al., 1996: L-shaped system, transient vibration, time-domain analysis), (Vardy et al., 1996: T-shaped system, transient vibration, time-domain analysis) and (Tijsseling and Vaugrante, 2001: L- and T-shaped systems, free vibration, frequency-domain analysis). Fan and Tijsseling (1992) and Tijsseling et al. (1996) presented experimental results for FSI with cavitation in straight and L-shaped pipes, respectively. The present paper completes the series of FSI/cavitation experiments with the T-shaped pipe.

\section{Laboratory apparatus}

The T-piece pipe is shown in Fig. 1. It consists of a $4.51 \mathrm{~m}$ long pipe (pipe 1) connected through a rigid $\mathrm{T}$-junction to two $1.34 \mathrm{~m}$ short pipes (pipes 2 and 3). The pipes are made of stainless steel and have 
an inner diameter of $52 \mathrm{~mm}$ and a wall thickness of $3.945 \mathrm{~mm}$. They are closed at their remote ends and filled with pressurised ordinary tap water. The system is symmetrical and is suspended in a horizontal plane. It hangs on four long, thin, vertical, adjustable, steel wires, which allow horizontal vibration without significant restraint. The system is excited by the external impact of a $5 \mathrm{~m}$ long solid steel rod, also hanging on wires. In the experiments reported herein, the rod hits the end plug axially, i.e. the central axes of the rod and the long pipe are in one line at the instant of impact. Table 1 gives the material and geometrical properties of the system. Fig. 2 shows the impact end and Fig. 3 shows the T-junction.

The T-piece pipe is instrumented with piezo-electric pressure transducers ("pt") at 8 locations and strain gauges ("sg") at 6 locations, as indicated in Fig. 1. The pressure transducers are positioned along the bottoms of the pipes and at the centres of the end caps. Each transducer (Kistler 7031) records the transient dynamic pressure and one transducer (Kistler 4073A100) also records the initial and final static pressures. The strain gauges record the dynamic axial strains at the top, bottom and sides of the pipes. The sensed signals are amplified and fed into two data acquisition systems (with, in total, 22 channels) at sampling rates up to a maximum of $1 \mathrm{MHz}$ per channel.

An essential element of the experiment is the lining out of the system. This is done with an estimated accuracy of two tenths of a millimetre. A surveying level and staff are used to confirm the system's horizontal alignment and a theodolite is used when bringing the impact rod and the long pipe into one vertical plane. The proper relative vertical elevation of the rod and pipe is checked with a dial gauge. The system is held in its stationary position by remote-controlled magnets that have negligible influence on the subsequent dynamic behaviour.

A small valve in the end plug enables changing the static pressure in the system by means of a hand pump. The static pressure controls the occurrence and severity of cavitation in the water.

This physical experiment has the important advantages of (i) no unknown support conditions, (ii) clearly defined excitation, and (iii) no gaseous cavitation in the transient event. Gaseous cavitation is a diffusive process with an "incubation time" of several seconds (for water at room temperature) (Zielke et al., 1989), which is long compared to the time scale of the experiment (milliseconds). Furthermore, the influence of gas is much reduced by pressurising the water directly after filling. One minor disadvantage for the cavitation experiments is the strong temperature-dependence of the static pressure. The water temperature, which itself was not measured, could be deduced from the static pressure. 


\section{Experimental results}

In theory, the T-shaped pipe and the excitation are symmetrical about the central axis of the long pipe. This means that the long pipe should vibrate in its axial direction only, i.e. in-plane and out-of-plane flexural vibration should not occur. The axial motion of the long pipe induces lateral motion of the short pipes. The pressure variations in the liquid cause axial motion of the short pipes. Cavitation occurs when the pressure falls to vapour pressure, which is about $0.002 \mathrm{MPa}$ for water at room temperature.

Figs. 4 and 5 show absolute transient pressures measured close to the T-junction for different static pressures. Fig. 4 shows pressures measured in two subsequent (typically five-minutes time gap) experimental runs under practically the same conditions. Fig. 5 shows pressures measured on both sides of the T-junction.

Figs 4(a) and 5(a) display experiments without cavitation. The initial (static) pressure $P_{0}$ is $2 \mathrm{MPa}$, which is sufficiently high to prevent cavitation occurring at any location in the system. Fig. 4(a) shows that the repeatability of the experiment is excellent and Fig. 5(a) confirms the symmetry of the system; in both figures, two different lines are drawn, but these are not distinguishable at this plotting scale.

The axial impact of the rod onto the closed end of the long pipe generates a pressure wave in the liquid and a longitudinal stress wave in the pipe wall. The front of the stress wave arrives at the T-junction about $1 \mathrm{~ms}$ after impact and it makes the T-junction move suddenly. This movement results in a pressure drop according to the Joukowsky relation $\Delta P=\rho c \Delta V$, where the jumps in pressure $(\Delta P)$ and velocity $(\Delta V)$ are proportional; the factor $\rho c$ is the product of the liquid's mass density and its effective sonic speed. The compressive pressure wave generated by the rod impact needs $3.4 \mathrm{~ms}$ to reach the T-junction. This sudden pressure rise makes the T-junction move. A detailed analysis and interpretation of the measurements is practicable only with the aid of a dedicated computer code (Vardy et al., 1996).

The Figs. 4(b) and 5(b) show measured pressures for a decreased initial (static) pressure $P_{0}$ of 0.90 MPa. The signals differ from those in the Figs. 4(a) and 5(a) because of cavitation in the liquid. At some point in the system (but not at "pt5" and "pt7", see below), the pressure has reached the vapour pressure. The signals indicate that cavitation has not occurred near the T-junction itself: the pressure histories are 
well above the zero line.

The Figs. 4(c,d) and 5(c,d) show measured pressures for further decreased initial pressures $P_{0}$ of 0.70 $\mathrm{MPa}$ and $0.50 \mathrm{MPa}$, respectively. The pressure signals touch the zero line, so some cavitation might have occurred at the T-junction.

The Figs. 4(e,f) and 5(e,f) show measured pressures for low initial pressures $P_{0}$ of $0.30 \mathrm{MPa}$ and 0.10 $\mathrm{MPa}$, respectively. The liquid cannot sustain the first potential pressure drop of $0.5 \mathrm{MPa}$ and a void will develop at the T-junction. The pressure remains at the vapour pressure of $0.002 \mathrm{MPa}$ until the void collapses. In this instance, the void-collapse, like the rod-impact, gives a steep rise in pressure.

It is evident that the occurrence of cavitation has a negative effect on the repeatability and symmetry of the experiment. This is due to the random nature of cavitation and its dependence on the system's static pressure, which itself is highly sensitive to the temperature.

Fig. 6 shows the pressures at the remote ends of pipes 2 and 3. The lateral motion of these short pipes has no direct effect on the mean pressure, so the first pressure drop is due to the low-pressure wave originating at the T-junction and arriving at the far ends about $1.3 \mathrm{~ms}$ after impact. Because the ends are closed, the pressure reduction becomes twice as large upon reflection, namely about 1 MPa. Fig. 6(b) shows that an initial pressure $P_{0}$ of $0.90 \mathrm{MPa}$ is not sufficient to sustain this pressure drop, and so it is likely that the closed ends of the short pipes are the locations where cavitation occurs first (for $P_{0}$ larger than 0.50 MPa, otherwise cavitation starts elsewhere). As already observed in previous experiments, short-duration tensile stresses $(P<0)$ precede the first occurrence of cavitation.

Fig. 7 shows the bending strains in the short pipes. These are defined as the differences $\left(\varepsilon_{1}-\varepsilon_{3}\right) / 2$ in axial strain measured at opposite sides of the pipe circumference and they are directly related to the bending moments via $M=\left(\varepsilon_{1}-\varepsilon_{3}\right) E I /[2(R+e)]$, with $E I$ the flexural stiffness and $R+e$ the outer radius of the pipe. The flexural vibration of the short pipes is not much influenced by the contained liquid. The effect of the liquid is indirect through the changed axial vibration of the long pipe, this being the driver of flexure of the short pipes.

Fig. 8 shows the corresponding axial strains $\left(\varepsilon_{1}+\varepsilon_{3}\right) / 2$ in the short pipes. Axial vibration of the short pipes is caused by pressure variations in the water. 
Fig. 9 shows pressures near the impact plug measured in two subsequent runs. The rod impact generates a pressure rise of $0.77 \mathrm{MPa}$ magnitude. Cavitation is observed in all five figures (b)-(f). It is noted that the pressure transducer ("pt1" in Fig. 1) is $20 \mathrm{~mm}$ away from the closed end and may be close to the edge of the column separations occurring there. All signals measured at this location, also with different transducers and different types of mounting, showed spurious oscillations and unrealistic spikes. In fact, several transducers were damaged, some with a hole in the diaphragm. Apparently, the local, nearby explosion and implosion of small cavitation bubbles is a too severe load for them.

Most interesting is Fig. 9(f) from which it is clearly seen that cavitation starts almost immediately after impact. The initial static pressure is so low here that the radial expansion of the pipe wall (accompanying the compressive axial stress wave) induces a pressure drop sufficiently high to cause cavitation.

\section{Conclusions}

1. Detailed measurements of FSI and vaporous cavitation have been presented for a laboratory apparatus including three pipes in an horizontal plane. All three pipes share a common junction (a Tpiece) and their remote ends are closed.

2. The apparatus is nominally symmetrical and so is the excitation, namely a controlled, axial impact at the remote end of the longest pipe. In theory, identical results should be obtained in pipes 2 and 3, and on opposite faces of pipe 1. In practice, minor differences are observed, but a high degree of symmetry is exhibited even in cases involving significant degrees of cavitation.

3. The measurements have been shown to be highly reproducible. This is a necessary requirement of any measurements designed expressly for software validation purposes. It is especially noteworthy in the case of successive tests involving vaporous cavitation of ordinary tap water.

4. The data are believed to be sufficiently accurate, sufficiently detailed and sufficiently complete to justify their widespread use for the assessment of theoretical models of FSI and cavitation. Accordingly, the data will be made freely available to the international community through a website at Eindhoven University of Technology with financial support from the Surge-Net project: www.win.tue.nl/fsi. 


\section{Acknowledgements}

The Surge-Net project (www.surge-net.info) is supported by funding under the European Commission's Fifth Framework 'Growth' Programme via Thematic Network "Surge-Net” contract reference: G1RTCT-2002-05069. The authors of this paper are solely responsible for its content, which might not reflect the opinion of the Commission. The Commission is not responsible for any use that might be made of data herein.

Colin Stark, Della Leslie and Ernie Kuperus are thanked for their skills and technical assistance in running the experiments.

\section{References}

Fan, D., Tijsseling, A., 1992. Fluid-structure interaction with cavitation in transient pipe flows. ASME Journal of Fluids Engineering 114, 268-274.

Tijsseling, A.S., 1996. Fluid-structure interaction in liquid-filled pipe systems: a review. Journal of Fluids and Structures 10, 109-146.

Tijsseling, A.S., Vardy, A.E., Fan, D., 1996. Fluid-structure interaction and cavitation in a single-elbow pipe system. Journal of Fluids and Structures 10, 395-420.

Tijsseling, A.S., Vaugrante, P., 2001. FSI in L-shaped and T-shaped pipe systems. In: Brekke, H., Kjeldsen, M. (Eds.), Proceedings of the 10th International Meeting of the IAHR Work Group on the Behaviour of Hydraulic Machinery under Steady Oscillatory Conditions, Trondheim, Norway, Paper C3.

Vardy, A.E., Fan, D., 1989. Flexural waves in a closed tube. In: Thorley, A.R.D. (Ed.), Proceedings of the 6th International Conference on Pressure Surges, BHRA, Cambridge, UK, 43-57.

Vardy, A.E., Fan, D., Tijsseling, A.S., 1996. Fluid/structure interaction in a T-piece pipe. Journal of Fluids and Structures 10, 763-786. 
Wiggert, D.C., Tijsseling, A.S., 2001. Fluid transients and fluid-structure interaction in flexible liquid-filled piping. ASME Applied Mechanics Reviews 54, 455-481.

Zhang, L., Tijsseling, A.S., Vardy, A.E., 1999. FSI analysis of liquid-filled pipes. Journal of Sound and Vibration 224(1), 69-99.

Zielke, W., Perko, H.-D., Keller, A., 1989. Gas release in transient pipe flow. In: Thorley, A.R.D. (Ed.), Proceedings of the 6th International Conference on Pressure Surges, BHRA, Cambridge, UK, 3-13. 


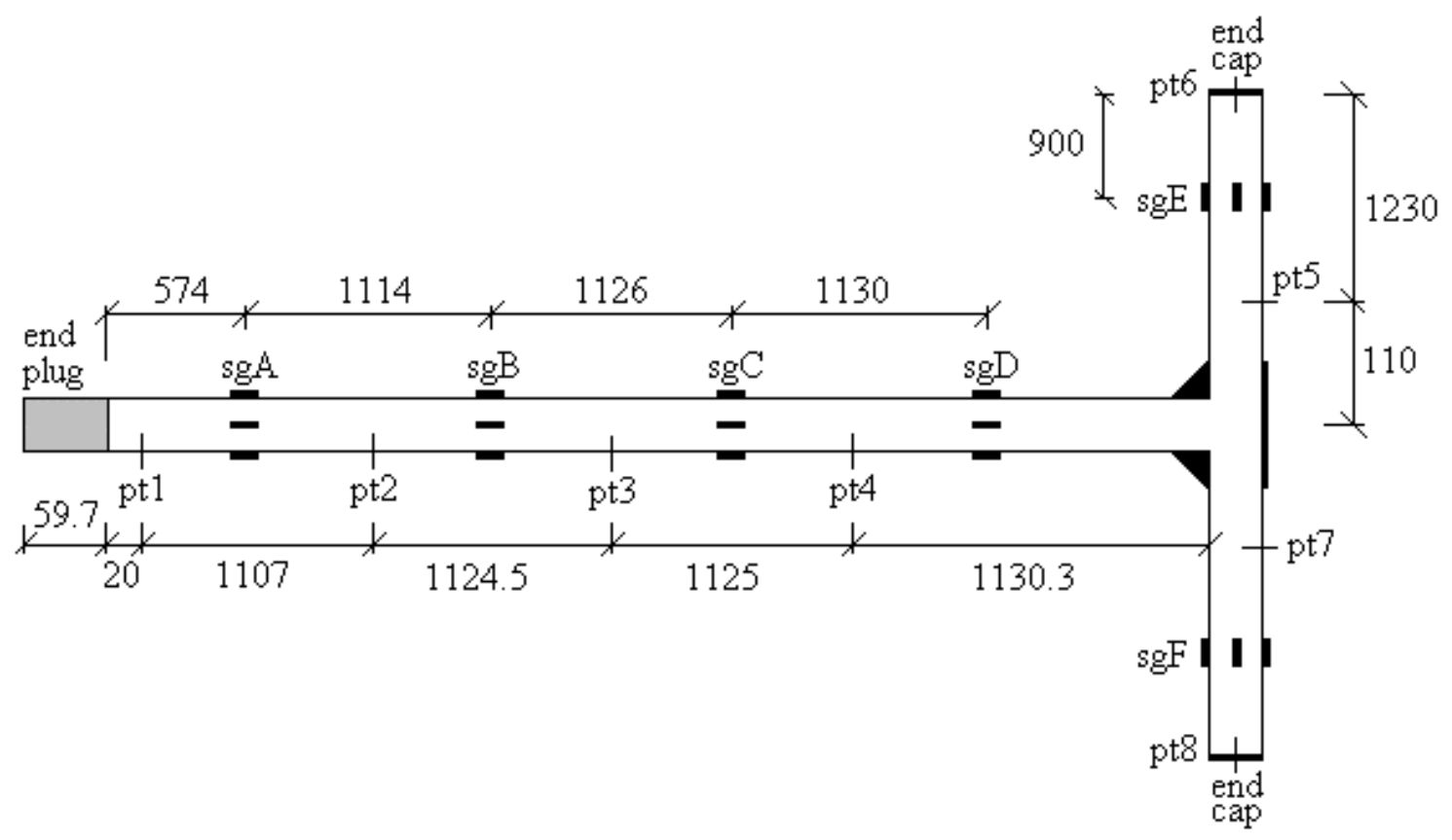

Fig. 1. Top view of the symmetric T-piece apparatus with lengths in mm (not to scale).

Table 1

Material and geometrical properties of the T-piece apparatus.

\begin{tabular}{cccc}
\hline & Steel pipes & Impact rod & Water \\
\hline Length(s) & $4.51 \mathrm{~m}, 1.34 \mathrm{~m}, 1.34 \mathrm{~m}$ & $5.006 \mathrm{~m}$ & \\
Radius & $26.01 \mathrm{~mm}$ (inner) & $25.37 \mathrm{~mm}$ & \\
& $29.96 \mathrm{~mm}$ (outer) & & \\
Young modulus & $168 \mathrm{GPa}$ & $200 \mathrm{GPa}$ & $999 \mathrm{~kg} / \mathrm{m}^{3}$ \\
Mass density & $7985 \mathrm{~kg} / \mathrm{m}^{3}$ & $7848 \mathrm{~kg} / \mathrm{m}^{3}$ & $2.14 \mathrm{GPa}$ \\
Bulk modulus & & & \\
Poisson ratio & 0.29 & $0.809 \mathrm{~m} / \mathrm{s}$ & \\
Impact velocity & & T-junction & End cap \\
\hline Mass & Impact plug & $1.06 \mathrm{~kg}$ & $0.32 \mathrm{~kg}$
\end{tabular}




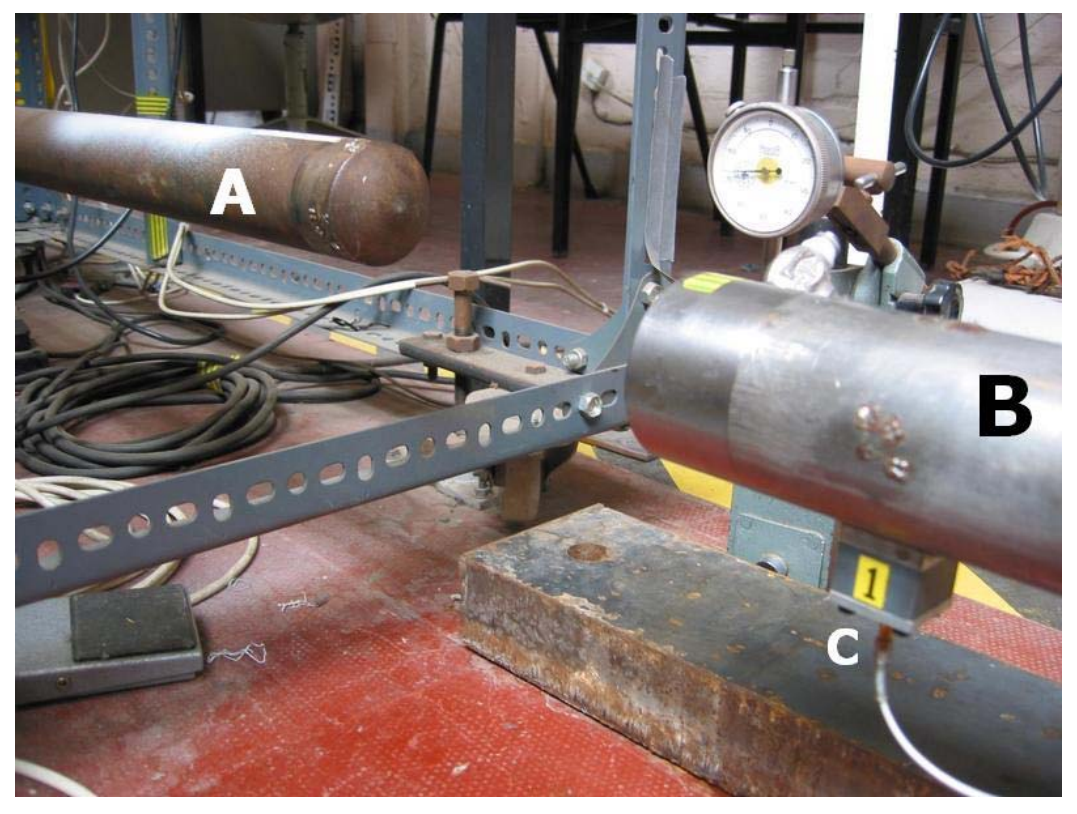

Fig. 2. Impact rod (A), long pipe (B) and pressure transducer "pt1" (C).

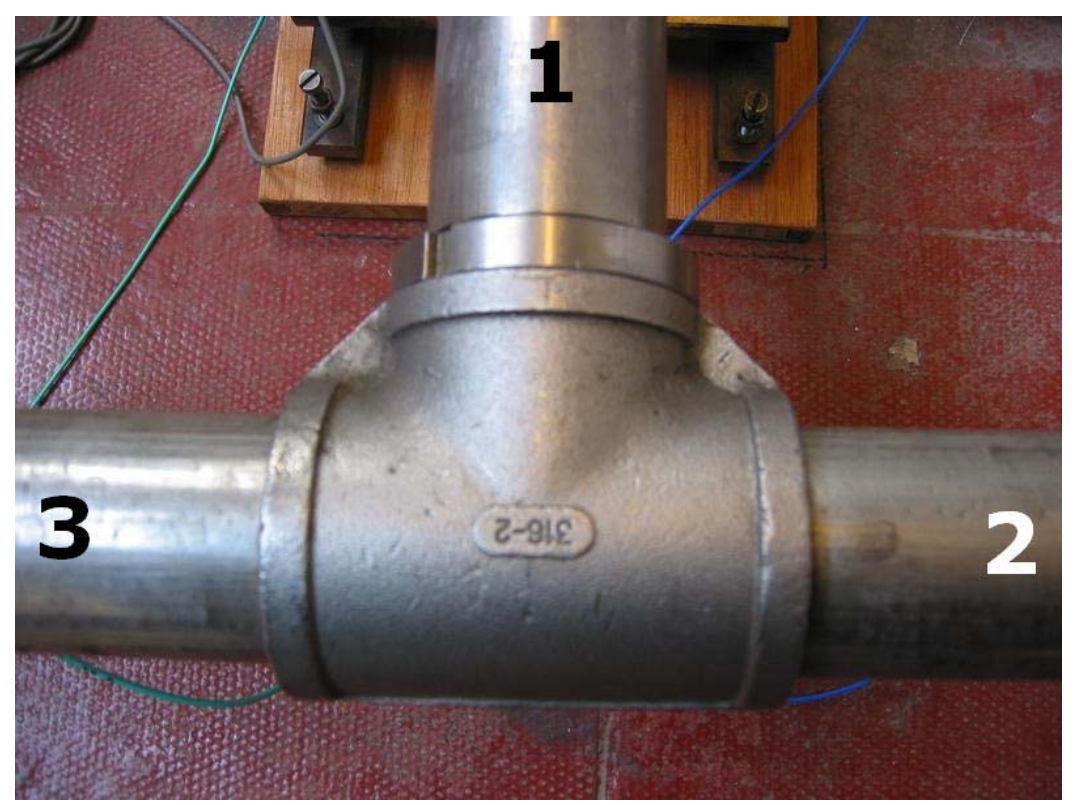

Fig. 3. T-junction connecting the pipes 1,2 and 3. 


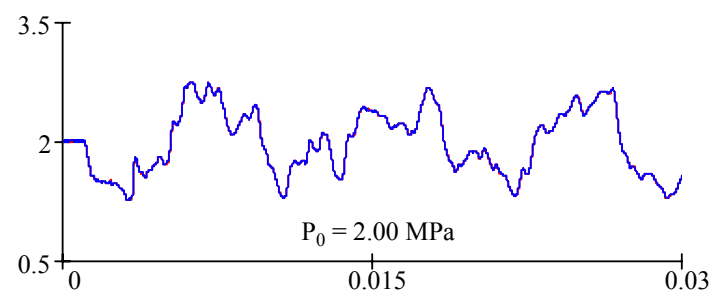

(a)

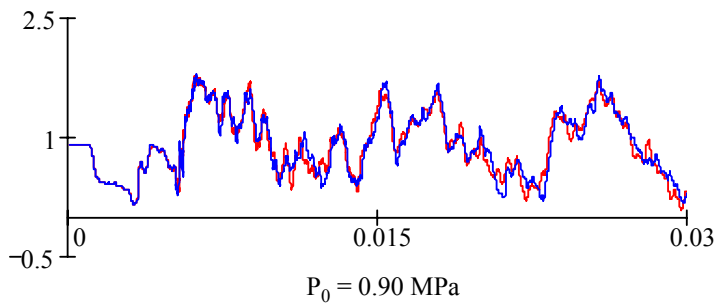

(b)

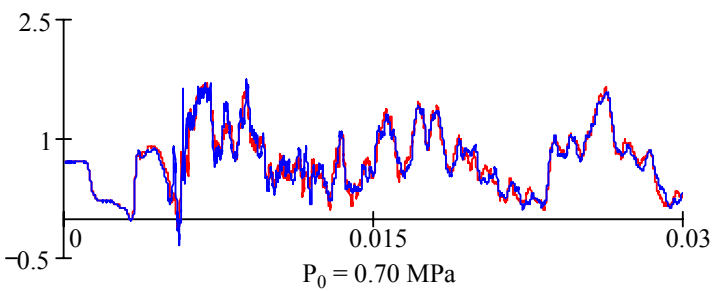

(c)

Fig. 4. Repeatability of the experiment for six different static pressures. In each figure, two lines are drawn, corresponding to two different tests under practically the same conditions. Transient pressures measured close to the Tjunction at location "pt7" (in Fig. 1) are shown. The initial static pressure $P_{0}$ ranges from $2 \mathrm{MPa}$ (and no cavitation) in figure (a) to 0.1 MPa (and much cavitation) in figure (f). Horizontal axis: time (s). Vertical axis: absolute pressure $(\mathrm{MPa})$.

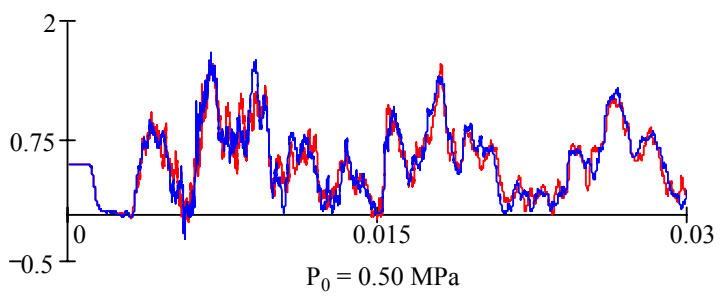

(d)

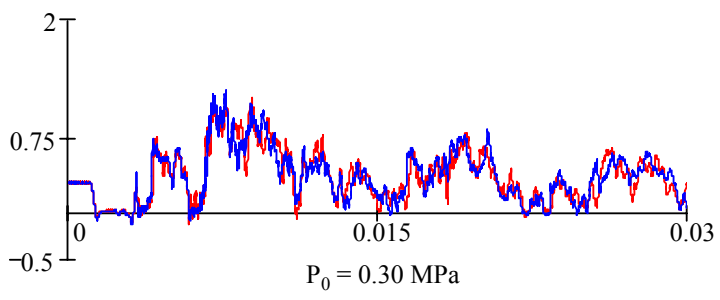

(e)

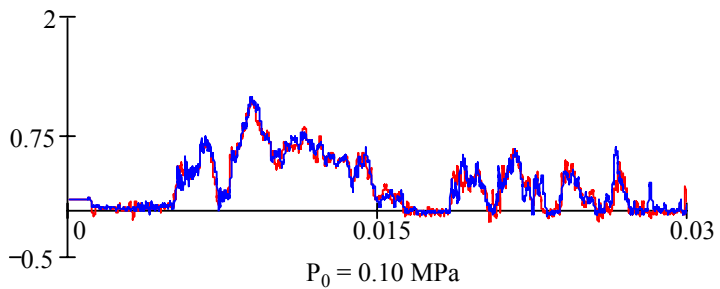

(f) 


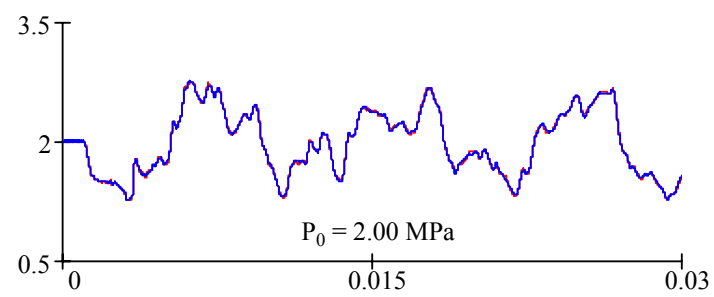

(a)

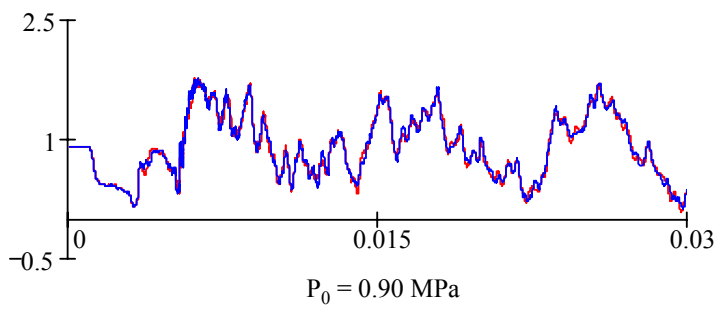

(b)

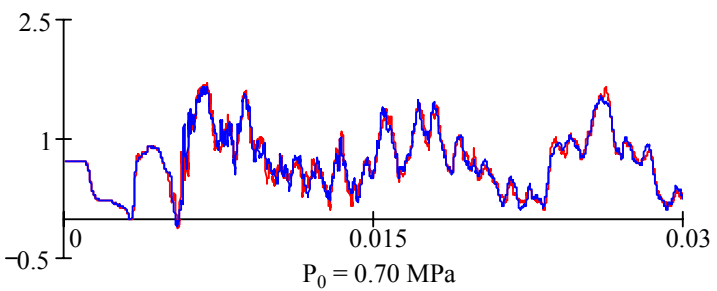

(c)

Fig. 5. Symmetry of the experiment for six different static pressures. In each figure, two lines are drawn, corresponding to transient pressures measured close to the T-junction at the opposite locations "pt5" and "pt7" (in Fig. 1). The initial static pressure $P_{0}$ ranges from $2 \mathrm{MPa}$ (and no cavitation) in figure (a) to $0.1 \mathrm{MPa}$ (and much cavitation) in figure (f). Horizontal axis: time (s). Vertical axis: absolute pressure (MPa).

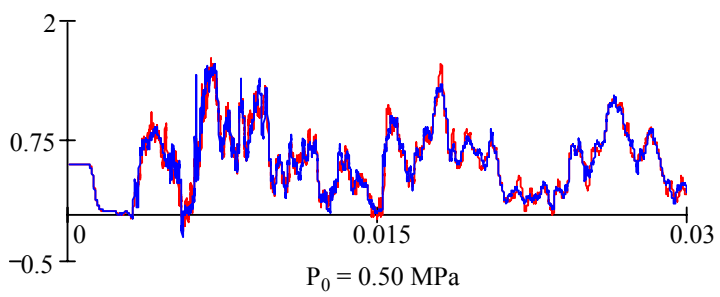

(d)

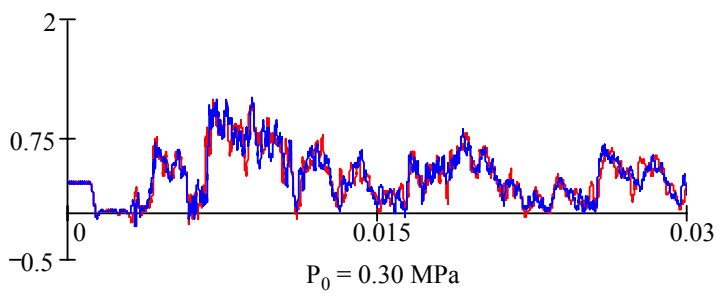

(e)

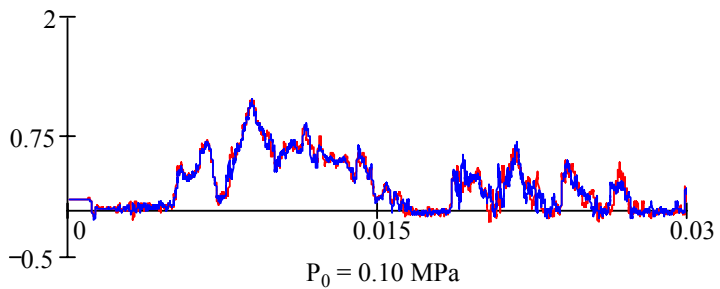

(f) 


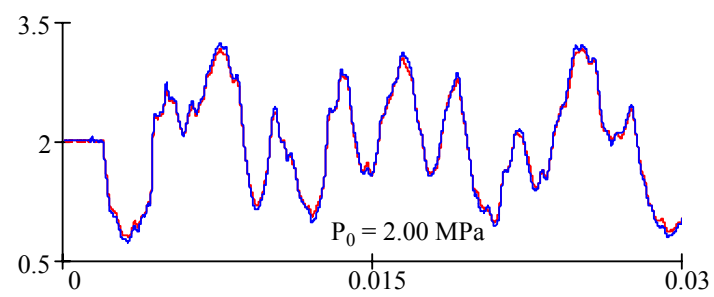

(a)

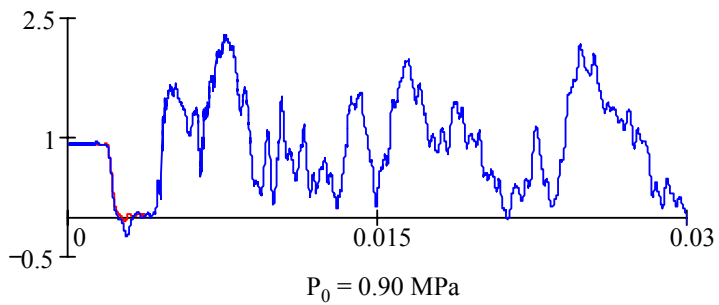

(b)

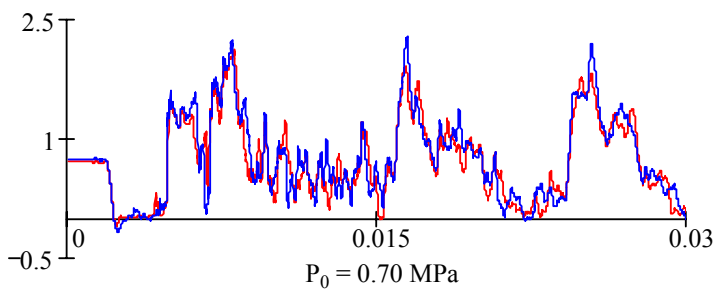

(c)

Fig. 6. Symmetry of the experiment for six different static pressures. In each figure, two lines are drawn, corresponding to transient pressures measured close to the remote ends at the opposite locations "pt6" and "pt8" (in Fig. 1). The initial static pressure $P_{0}$ ranges from $2 \mathrm{MPa}$ (and no cavitation) in figure (a) to $0.1 \mathrm{MPa}$ (and much cavitation) in figure (f). Horizontal axis: time (s). Vertical axis: absolute pressure (MPa).

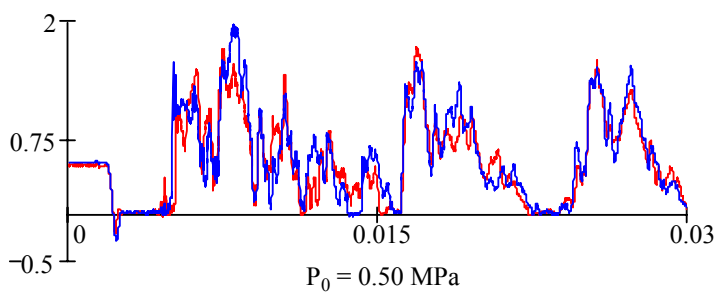

(d)

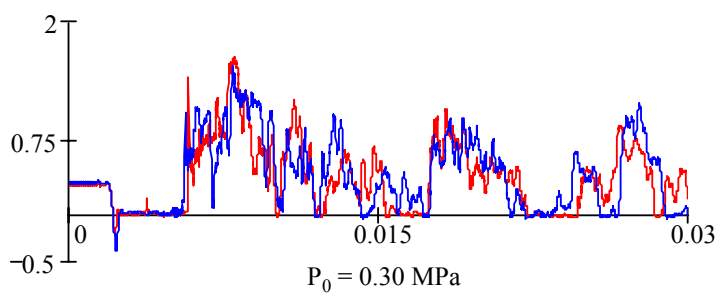

(e)

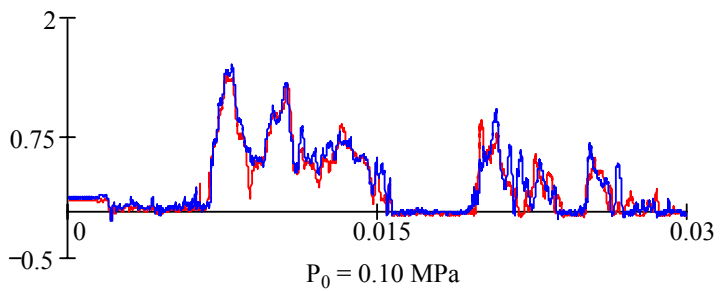

(f) 


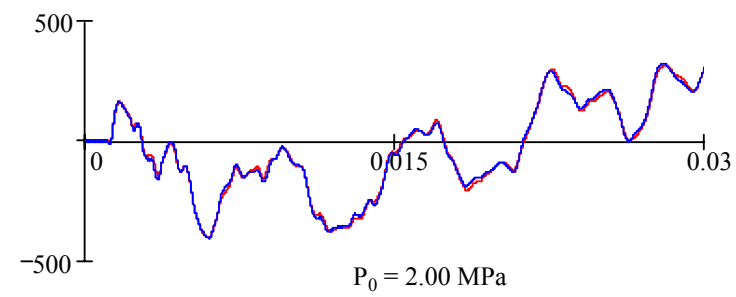

(a)

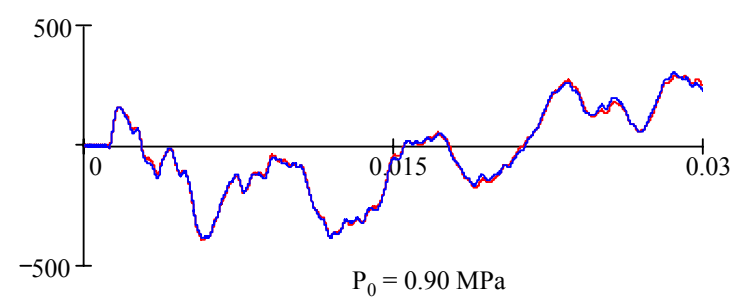

(b)

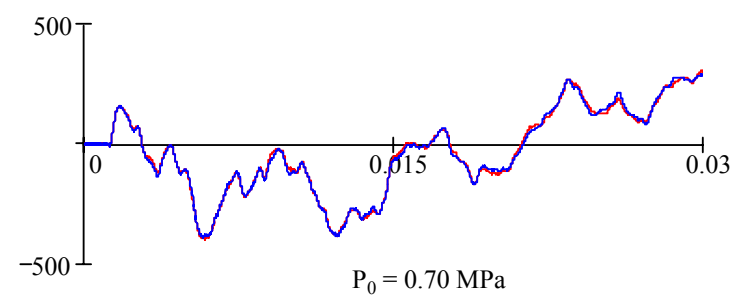

(c)

Fig. 7. Symmetry of the experiment for six different static pressures. In each figure, two lines are drawn, corresponding to bending strains measured in the short pipes at the opposite locations "sgE" and "sgF" (in Fig. 1). The initial static pressure $P_{0}$ ranges from $2 \mathrm{MPa}$ (and no cavitation) in figure (a) to $0.1 \mathrm{MPa}$ (and much cavitation) in figure (f). Horizontal axis: time (s). Vertical axis: bending strain (micro-strain).

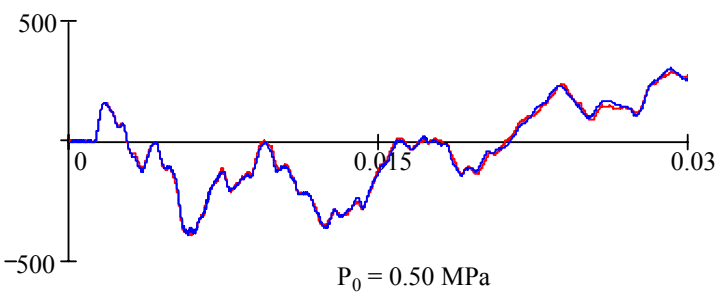

(d)

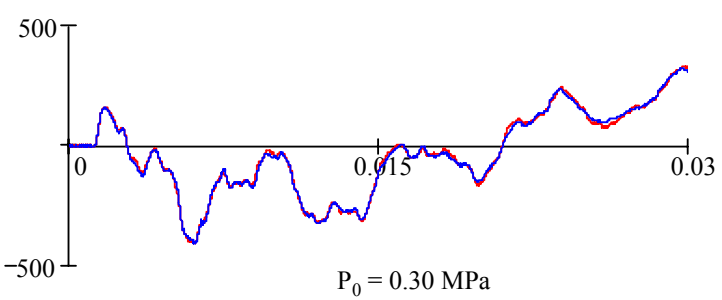

(e)

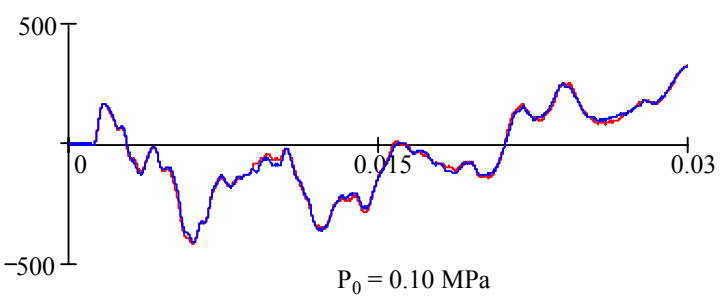

(f) 


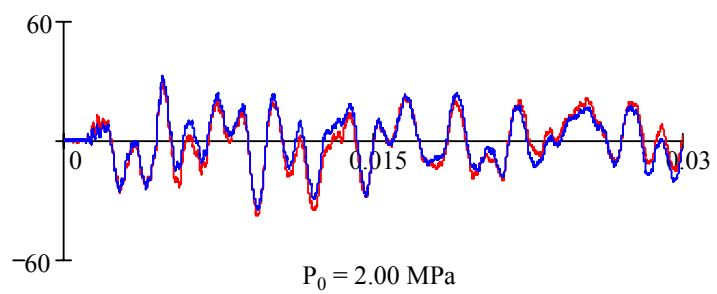

(a)

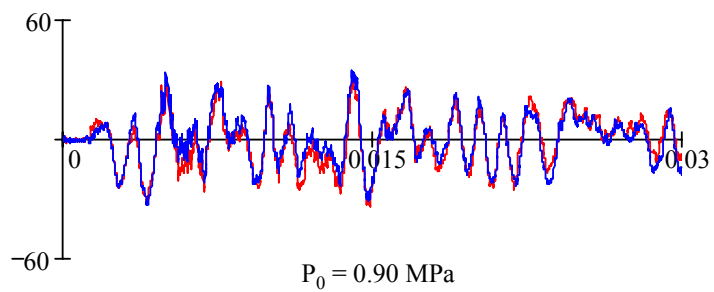

(b)

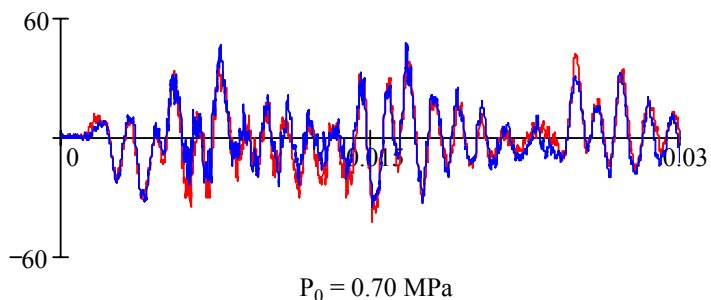

(c)

Fig. 8. Symmetry of the experiment for six different static pressures. In each figure, two lines are drawn, corresponding to axial strains measured in the short pipes at the opposite locations "sgE" and "sgF" (in Fig. 1). The initial static pressure $P_{0}$ ranges from $2 \mathrm{MPa}$ (and no cavitation) in figure (a) to $0.1 \mathrm{MPa}$ (and much cavitation) in figure (f). Horizontal axis: time (s). Vertical axis: axial strain (micro-strain).

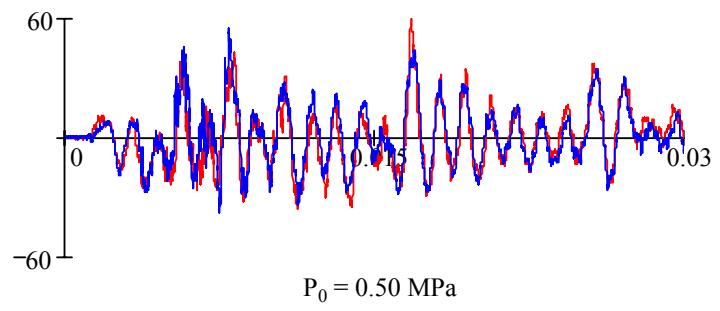

(d)

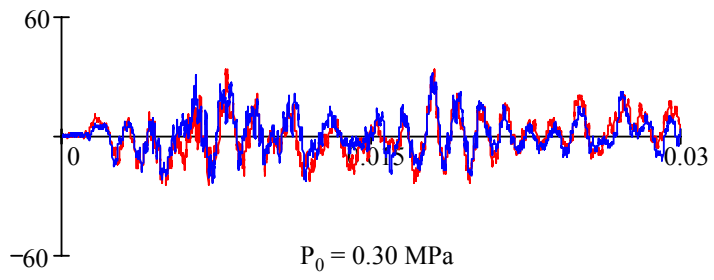

(e)

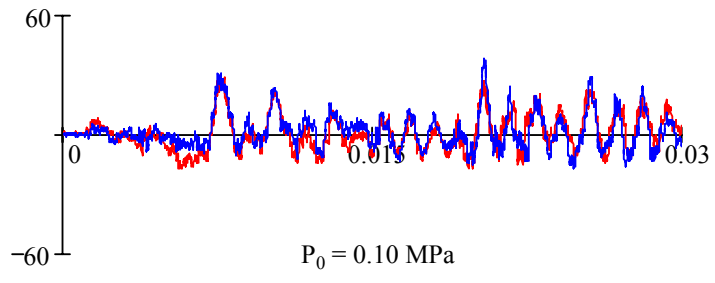

(f) 


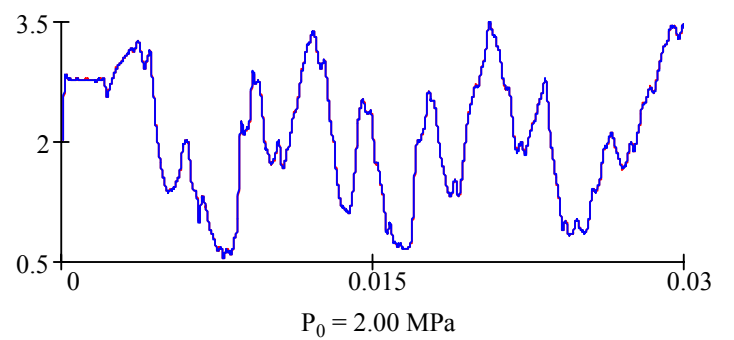

(a)

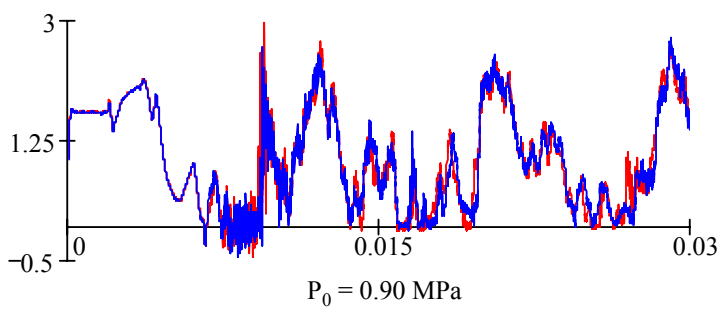

(b)

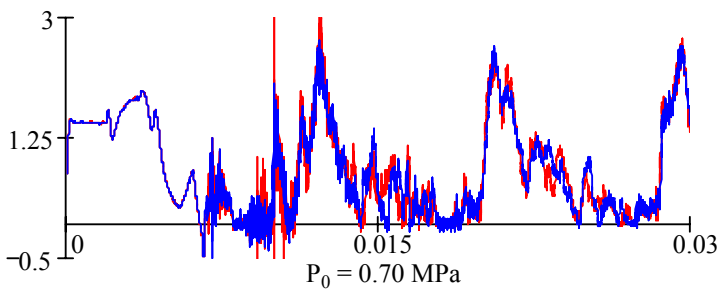

(c)

Fig. 9. Repeatability of the experiment for six different static pressures. In each figure, two lines are drawn, corresponding to two different tests under practically the same conditions. Transient pressures measured close to the impact end at location "pt1" (in Fig. 1) are shown. The initial static pressure $P_{0}$ ranges from $2 \mathrm{MPa}$ (and no cavitation) in figure (a) to $0.1 \mathrm{MPa}$ (and much cavitation) in figure (f). Horizontal axis: time (s). Vertical axis: absolute pressure $(\mathrm{MPa})$.

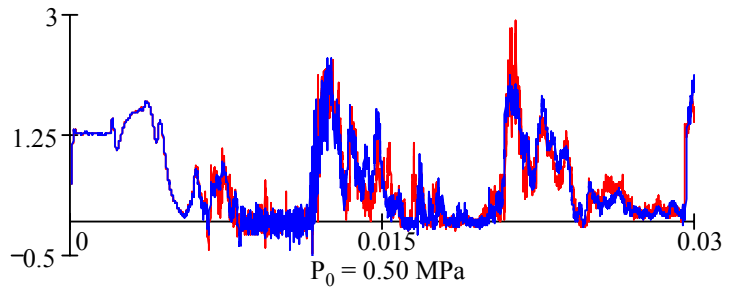

(d)

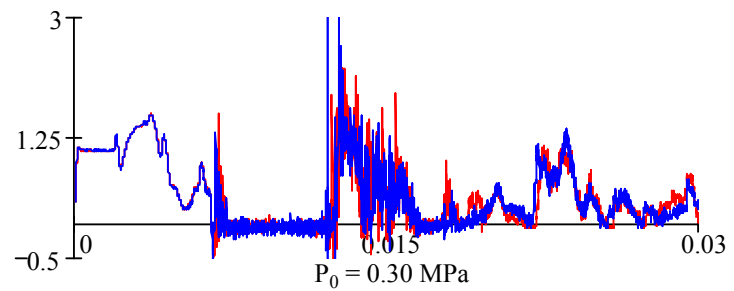

(e)

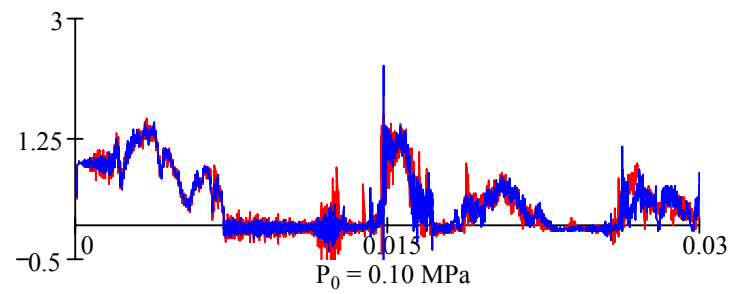

(f) 